\title{
A discovery of young stellar objects in older clusters of the Large Magellanic Cloud
}

\author{
Bi-Qing For, ${ }^{1 \star}$ and Kenji Bekki ${ }^{1}$ \\ ${ }^{1}$ International Centre for Radio Astronomy Research/The University of Western Australia, \\ M468, 35 Stirling Highway, Crawley, WA 6009, Australia
}

Accepted XXX. Received YYY; in original form ZZZ

\begin{abstract}
Recent studies have shown that an extended main-sequence turn-off is a common feature among intermediate-age clusters (1-3 Gyr) in the Magellanic Clouds. Multiplegeneration star formation and stellar rotation or interacting binaries have been proposed to explain the feature. However, it remains controversial in the field of stellar populations. Here we present the main results of an ongoing star formation among older star clusters in the Large Magellanic Cloud. Cross-matching the positions of star clusters and young stellar objects has yielded 15 matches with 7 located in the cluster center. We demonstrate that this is not by chance by estimating local number densities of young stellar objects for each star cluster. This method is not based on isochrone fitting, which leads to some uncertainties in age estimation and methods of background subtraction. We also find no direct correlation between atomic hydrogen and the clusters. This suggests that gas accretion for fueling the star formation must be happening in situ. These findings support for the multiple-generations scenario as a plausible explanation for the extended main-sequence turn-off.
\end{abstract}

Key words: galaxies: star clusters: general — Magellanic Clouds

\section{INTRODUCTION}

Star clusters (SCs) are fundamental building blocks of galaxies. Their physical properties encode valuable information not only on their own formation processes but also on the evolutionary histories of their host galaxies. They were once thought to form in a single epoch of star formation that produced thousands or millions of coeval stars with the same chemical composition. Recent high-quality Hubble Space Telescope (HST) photometric studies of colormagnitude diagrams (CMDs) of intermediate-age (1-3 Gyr) SCs in the Magellanic Clouds uncovered evidence of multiple stellar populations with unexpected characteristics, such as two distinct main-sequence turn-off for NGC 1846 (Mackey \& Broby Nielsen 2007). Some of the Galactic globular clusters also reveal multiple stellar populations with different helium and light element abundances (e.g., see Piotto et al. 2015; Milone et al. 2016). NGC 2808 is the most extreme cases where this phenomenon was first discovered (Piotto et al. 2007). These important discoveries have challenged the traditional view of SCs being formed in a single star formation episode (Mackey et al. 2008; Goudfrooij et al. 2015; Bekki \& Mackey 2009; Milone et al. 2015).

^ E-mail: biqing.for@uwa.edu.au
The direct explanation for the extended main-sequence turn-off (eMSTO) is that these clusters experienced prolonged star formation for 100-500 Myr (Goudfrooij et al. 2009, 2014), though one Large Magellanic Cloud (LMC) cluster (NGC 1783) has recently been reported to have a possible age spread of $\sim 1 \mathrm{Gyr}$ ( $\mathrm{Li}$ et al. 2016). These results suggest that LMC clusters that are $\lesssim 1$ Gyr old might still show signs of ongoing star formation today. However, such age spreads have recently been disputed by other observational and theoretical studies ( $\mathrm{Li}$ et al. 2014; Bastian et al. 2013). Stellar rotation or interacting binaries that can mimic an age apread on the CMD have been proposed as an alternative explanation (Bastian \& de Mink 2009; Yang et al. 2013; D'Antona et al. 2015 and references therein). It therefore remains unclear if the clusters contain multiple generations of stars.

To test the prolonged star formation scenario, we adopt a method that does not rely on isochrone fitting to the CMDs of the clusters, for which results have been refuted due to field star contamination in some cases (see e.g., Cabrera-Ziri et al. 2016). Young stellar objects (YSOs) are stars in their very early stage and have ages of less than 1 Myr (Dunham et al. 2015) so that the detection of YSOs can be considered as evidence of ongoing star formation. We therefore search for YSOs in the clusters with ages in the 
range of 0.1 to 1 Gyr in the LMC to determine if SCs could host multiple generations of stars.

In this letter, we report the finding of YSOs in the center regions of several older SCs. Our finding provides clear evidence for ongoing star formation in SCs that once completed their star formation, and therefore demonstrates the presence of multiple generations of stars in at least some LMC clusters.

\section{ANALYSIS AND RESULTS}

We employ the HERITAGE band-matched (Seale et al. 2014) and star cluster (Glatt et al. 2010) catalogues (hereafter S14 and G10) for the analysis. The S14 catalogue consists of astronomical objects that are detected in multiple HERITAGE images. These objects were positionally cross-matched and then further matched to Spitzer IRAC and MIPS point sources catalogues. The S14 catalogue is dominated by bright YSOs but potentially contaminated by background galaxies at low flux level. The S14 have employed step-by-step cuts to eliminate contamination. They also used Herschel photometry constraints on dust mass for asymptotic giant branch (AGB) progenitors to distinguish ambigious classification between post-AGB and YSOs. To take into account the flux confusion limit, YSOs were classified as having either a high probable or a moderate possible likelihood of being a YSO. The S14 catalogue employed here contains at most $1 \%$ evolved stars.

The G10 catalogue lists the derived ages and V-band luminosities of young SCs ( 9 Myr to $1 \mathrm{Gyr}$ ) in the Magellanic Clouds. The G10 study selected SCs from a general catalogue of extended objects in the Magellanic System (Bica et al. 2008; hereafter B08) and employed the photometric data of the Magellanic Clouds Photometric Survey (MCPS; Zaritsky et al. 2002, 2004). Double or multiple clusters classification (Dieball et al. 2002) is also adopted in the G10 catalogue.

We positionally cross-match the 2493 probable and 1025 possible YSOs listed in the S14 catalogue with the 738 SCs of ages in the range of 0.1 to 1 Gyr in the G10 catalogue. We adopt a search radius of $10 \mathrm{pc}$, which is a typical cluster size (Piatti et al. 2014). This search radius corresponds to an angular size of $41.4^{\prime \prime}$ by assuming the distance of the LMC to be $50 \mathrm{kpc}$. This radius takes into account the uncertainties discussed in the following sections.

We find 15 probable and 6 possible YSOs falling within the search radius. Two of these could also be post-AGB stars based on the classification in the S14 catalogue. In this Letter, we focus on the 15 probable YSOs, although the possible YSOs could also be genuine. Figure 1 shows the locations of the YSO detections in the LMC and Table 1 summarizes the cross-matching results and physical parameters of the SCs. Five out of fifteen young stellar candidates are confirmed to be genuine by photometric and spectroscopic observations, such as detection of ice and water masers (Gruendl \& Chu 2009; Sewiło et al. 2010). The other 10 are highly likely to be genuine YSOs being consistent with the spectral energy distributions and dust properties of YSOs (Seale et al. 2014). Among these 15 YSO candidates, 7 are located at the cluster center.

\subsection{Reliability}

The reliability of the cross-matching depends on the matching radius, completeness of the catalogues, source density and positional accuracy. The angular resolution and signalto-noise ratio of the data sets affect the positional accuracy. The S14 catalogue adopts the coordinates of point sources in the Herschel PACS $100 \mu \mathrm{m}$ point source catalogue if available. Herschel PACS $100 \mu \mathrm{m}$ images provide the highest angular resolution of all Herschel wavebands. If no detection is documented in the PACS $100 \mu \mathrm{m}$ point source catalogue, the coordinates of the next coarser angular resolution point source catalogue are adopted and so on. We examine the positional errors adopted in the S14 catalogue. The standard deviation of the errors is $0.1^{\prime \prime}$ with a median error of $0.06^{\prime \prime}$, which is very small as compared to the search radius.

Star cluster coordinates adopted from the B08 have an error of $10^{\prime \prime}-15^{\prime \prime}$, which corresponds to a maximum error of 4 pc. The apparent cluster size is generally underestimated due to visual inspection of the photographic plates. The search radius takes into account the positional accuracy of the SC and the uncertainty of the apparent cluster size. Nevertheless, we verify the matched objects by visual inspection using the Spitzer IRAC $3.6 \mu \mathrm{m}$ and Herschel PACS $100 \mu \mathrm{m}$ images for SCs and YSOs, respectively.

\subsection{Completeness}

There is a selection bias in the G10 catalogue. The catalogue only consists of well-defined and not too extended SCs. It is also limited to SCs with ages between $\sim 10 \mathrm{Myr}$ and 1 Gyr. Star clusters younger than 10 Myr are generally classified as associations or nebulae. Thus, they are excluded from the G10 catalogue. The upper age limit is due to the limiting photometric magnitude of the MCPS, resulting in the difficulty of resolving MSTO points of intermediate-age and older clusters. The S14 catalogue is complete for sources with fluxes brighter than 200 mJy in any single Herschel waveband over $>99 \%$ of the HERITAGE image area with a surface brightness of $<100 \mathrm{MJy} \mathrm{sr}^{-1}$ in the SPIRES $250 \mu \mathrm{m}$ waveband.

\subsection{Probability}

Fifteen SCs $\left(N_{\mathrm{S}-\mathrm{Y}}=15\right)$ have YSOs within $10 \mathrm{pc}$ of their centres. The total number of SCs $\left(N_{\mathrm{SC}}=738\right)$ in the G10 catalogue might be smaller than the actual number because of the limited sensitivity of the B08 and MCPS. Also, the total number of YSOs $\left(N_{\text {YSO }}=2493\right)$ in the S14 catalogue might be significantly smaller than that of all YSOs, because only bright YSOs have been detected. Therefore, $N_{\mathrm{S}-\mathrm{Y}}$ is likely to be underestimated. However, it is important for the present study to investigate whether or not the above $N_{\text {S-Y }}$ can be explained simply by a high probability of both a YSO and a SC being along the same line-of-sight (i.e., chance of alignment) and/or due to high local density of stars. We analytically estimate (i) the local probability for detecting the YSOs within 10 pc of SCs (i.e., search radius) based on the number of YSOs within the adopted $100 \mathrm{pc}$ $\left(N_{\mathrm{YSO}-100 \mathrm{pc}}\right)$ of $15 \mathrm{SCs}$, and (ii) $N_{\mathrm{YSO}-100 \mathrm{pc}}$ of $15 \mathrm{SCs}$ with respect to field star density. 

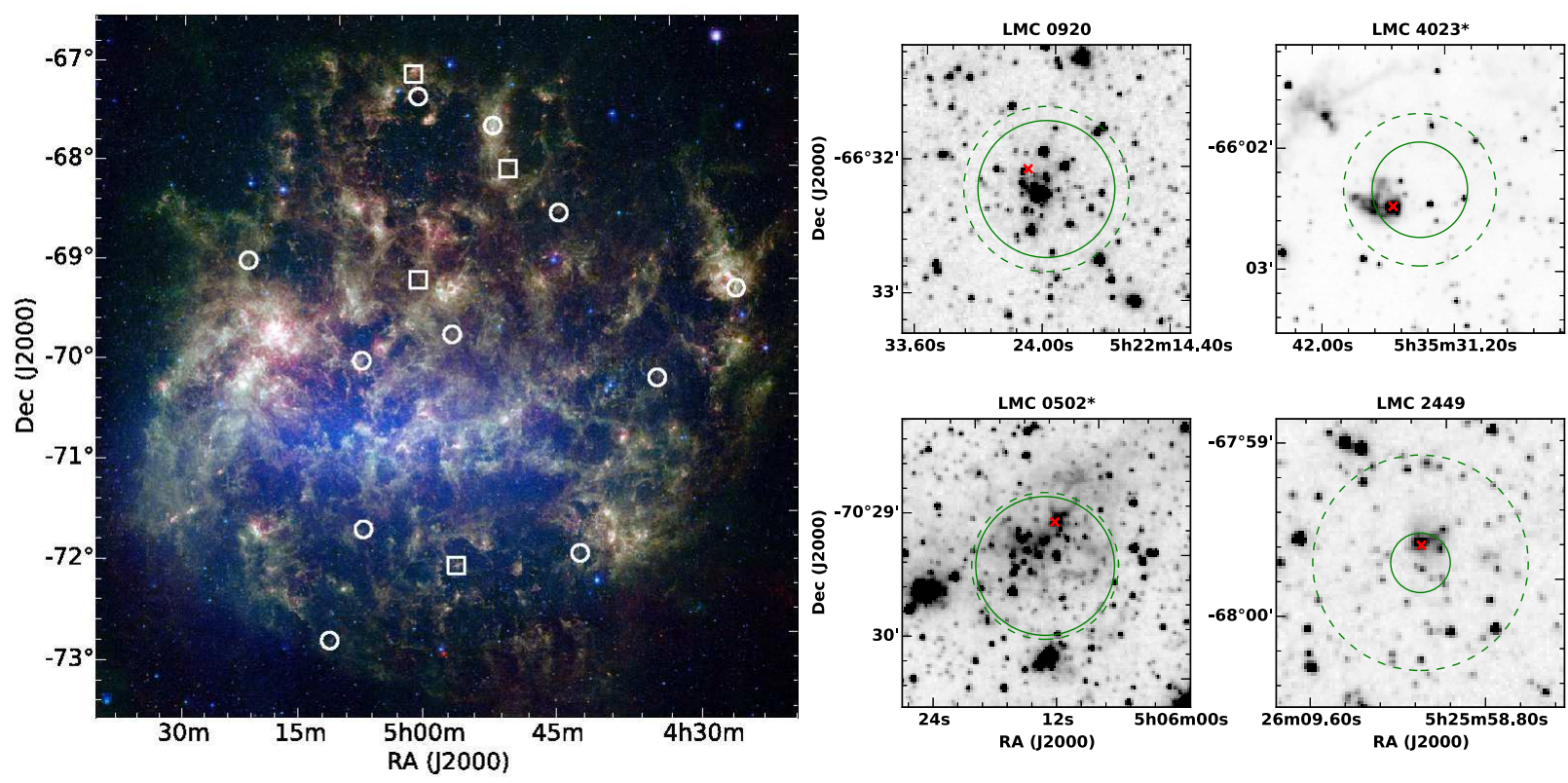

Figure 1. Detections of YSOs in the LMC clusters. Left: Circles and squares show the locations of 15 star clusters with detections of YSOs in the LMC. The clusters are overplotted onto a composite false-color image of the LMC from the Spitzer Surveying Agents of Galaxy Evolution survey (SAGE; Meixner et al. 2006). Squares represent the four star clusters shown in the right panel. Right: Examples of star clusters with detections of YSOs. The cluster images are 3.6 $\mu \mathrm{m}$ cutout images from the SAGE survey. The solid and dashed circles represent the apparent radii of the star cluster and the search radius of $10 \mathrm{pc}\left(41.4^{\prime \prime}\right)$, respectively. Crosses mark the locations of the YSOs. An asterisk in the cluster name indicates that the star cluster is a double/multiple system.

Table 1. Summary of cross-matching results and physical parameters of the star clusters with detections of young stellar objects

\begin{tabular}{lccccccccc}
\hline Cluster ID $^{a}$ & $\begin{array}{c}\text { Cluster RA } \\
\text { deg [J2000] }\end{array}$ & $\begin{array}{c}\text { Cluster Decl. } \\
\text { deg [J2000] }\end{array}$ & $\begin{array}{c}R_{\text {app }} \\
\text { arcmin }\end{array}$ & $\begin{array}{c}\log (\mathrm{age}) \\
\text { yr }\end{array}$ & $\begin{array}{c}V^{b} \\
\operatorname{mag}\end{array}$ & $\begin{array}{c}\text { YSO R.A. } \\
\operatorname{deg}[\mathrm{J} 2000]\end{array}$ & $\begin{array}{c}\text { YSO Decl. } \\
\operatorname{deg}[\mathrm{J} 2000]\end{array}$ & YSO classification $^{c}$ & $N^{d}$ \\
\hline LMC 0286 & 74.3583 & -67.6853 & 0.50 & 8.60 & 17.65 & 74.356865 & -67.675769 & defYSO/postAGB & 3 \\
LMC 0502* & 76.5875 & -70.4794 & 0.65 & 8.20 & 12.87 & 76.593826 & -70.472698 & probYSO \\
LMC 0848 & 79.9833 & -68.3500 & 0.16 & 8.00 & 15.71 & 79.960629 & -68.350753 & probYSO \\
LMC 0920 & 80.6208 & -66.5283 & 0.57 & 8.60 & 13.68 & 80.629594 & -66.526904 & probYSO \\
LMC 2228* & 79.0250 & -71.8069 & 0.16 & 8.00 & 16.64 & 79.014055 & -71.805417 & probYSO & 3 \\
\hline
\end{tabular}

Notes. Table 1 is published in its entirely as Supporting Information with the electronic version of the paper. A portion is shown here for guidance regarding its form and content. (a) Asterisk next to cluster name indicates possible double/multiple populations(Dieball et al. 2002). (b) $V$ magnitudes correspond to the total luminosity of all stars within $R_{\text {app }}$ (Glatt et al. 2010). (c) post-AGB: post Asymptotic Giant Branch star; defYSO: definite young stellar object; probYSO: high probability of being a young stellar object (Seale et al. 2014). (d) Number of YSOs within 100 pc of each star cluster.

The probability of a YSO being detected within the defined radius $(R)$ of a $\mathrm{SC}$ in the local YSO density $\left(P_{\mathrm{S}-\mathrm{Y}}\right)$ is $N_{\text {YSO-100pc }} \times(R / 100 \mathrm{pc})^{2}$. The mean probability for $R=10$, 3 and 1 pc are $5.6 \times 10^{-2}, 5.04 \times 10^{-3}$, and $5.6 \times 10^{-4}$, respectively, for $15 \mathrm{SCs}$ with YSOs. If we consider the inclination of the LMC $\left(i=27^{\circ}-45^{\circ}\right.$; van den Bergh 2000), then the projected surface area of the LMC is appreciably smaller but it does not affect $P_{\mathrm{S}-\mathrm{Y}}$. We note that 3 SCs (LMC 2442, LMC $2519^{*}$ and LMC 3829$)$ have a large $N_{\text {YSO-100pc }}(>10)$. Examining the spatial density of YSOs at 200 pc away from these 3 clusters, we only find a large $N_{\text {YSO-100pc }}$ for LMC 2519* and LMC 3829. This suggests that these two SCs are possibly in a very active star forming region and the probability for YSOs that are not physically associated to them is higher. Nevertheless, the low value of the mean probabilities demonstrates that detection of the other 13 SCs with YSOs is unlikely to be a chance coincidence but must be a real physical association of YSOs with the SCs.

By comparing the spatial density of YSOs in each of the 15 SCs with the density profile of the region, we can rule out the possibility that the detection of YSOs in these 15 clusters is due to high local field star density. This is because a higher detection rate would be expected in the inner region of the LMC, which is not the case. The density profile, $\Sigma D$, can be described as $\exp (-D / l)$, where $D$ is the distance between the SCs and the LMC centre and $l$ is the scale length of the LMC disc $(1.5 \mathrm{kpc})$. In Figure 2, we show the theoretical density profile (dashed line) and the number of YSOs within $100 \mathrm{pc}$ of SCs as a function of distance between the SCs and the LMC centre. There is no correlation. 


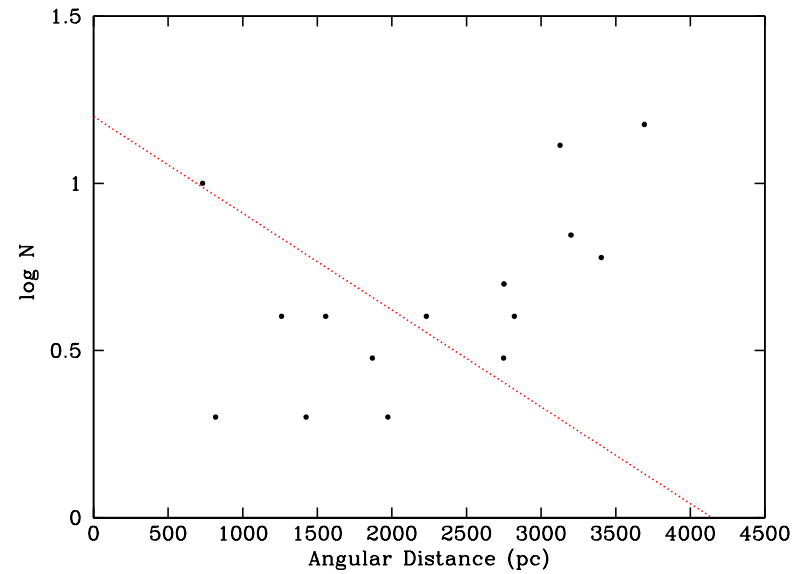

Figure 2. Angular distance between the SCs and the LMC centre vs. number of YSOs within 100 pc of 15 SCs on logarithmic scale. The dashed line corresponds to theoretical prediction of YSO number with respect to angular distance from the LMC centre with an arbitary zero-point for simplication.

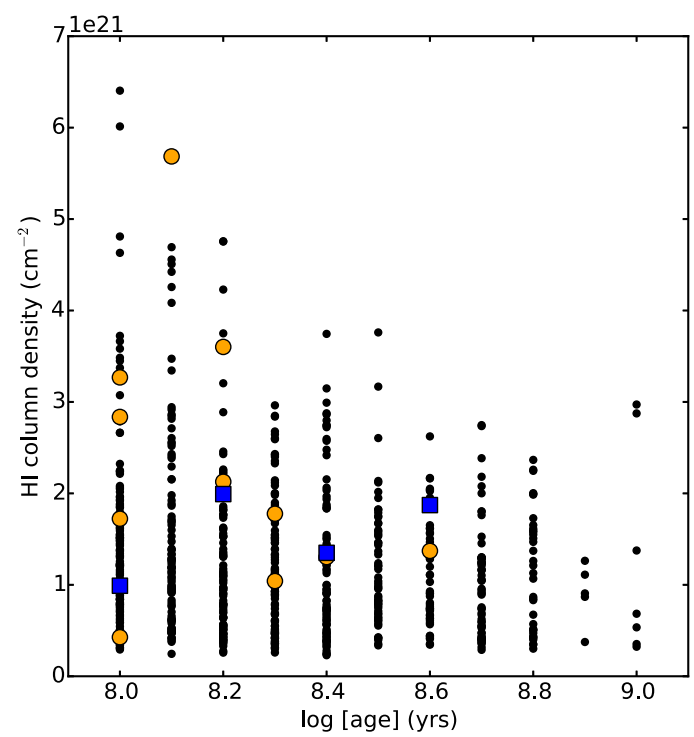

Figure 3. Mean HI column density within 10 pc of 738 star clusters vs. cluster age on a logarithmic scale. Star clusters with ages between 0.1 to $1 \mathrm{Gyr}$ are selected from the catalogue of young star clusters. Circles and squares correspond to the same symbols as shown in Figure 1. It is evident that the HI column density decreases with increasing cluster age. There is no direct correlation between $\mathrm{HI}$ gas and the star clusters with detection of YSOs.

\section{DISCUSSION AND CONCLUSIONS}

Accretion of interstellar atomic and/or molecular gas onto existing SCs can trigger second-generation star formation in the clusters (Bekki \& Mackey 2009; Li et al. 2016; Pflamm-Altenburg \& Kroupa 2009). We look for evidence of an external accretion event by investigating the atomic hydrogen gas (HI) content in the region of the SCs with identified YSOs using the combined single-dish (Parkes) and

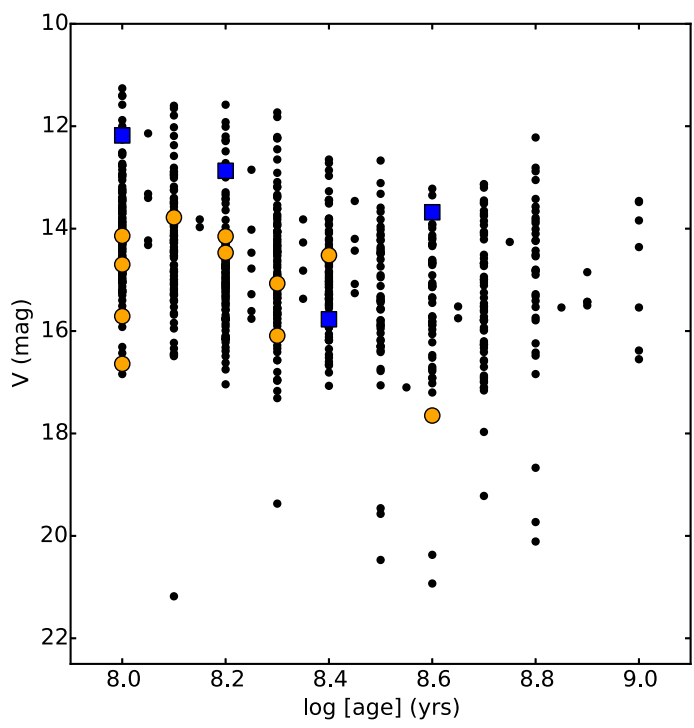

Figure 4. Luminosity ( $V$ mag) vs. age on a logarithmic scale of star clusters with ages between 0.1 to 1 Gyr. Data points and symbols represent the same as in Figure 1. There is no detection of YSOs in clusters older than 0.4 Gyr old.

interferometer (Australia Telescope Compact Array) LMC HI image (Kim et al. 2003). As a SC passes through a region of dense gas, accretion can occur and trigger star formation in situ. As shown in Figure 3, we find that there is no direct correlation between the $\mathrm{HI}$ gas and the clusters, which suggests that accretion of interstellar gas onto clusters is not responsible for the formation of YSOs in these clusters. However, we note that four SCs are situated at the rim of two HI supershells (SGS 7 and 11; Kim et al. 1999). These supershells are known to have a relatively high molecular gas concentration (Yamaguchi et al. 2001). Star formation can be triggered by shell expansion or accretion of the cold molecular gas. Interestingly, the detected YSOs in these four clusters reside in the cluster centre.

While our sample is small, we find that all SCs with YSOs are $\lesssim 0.4$ Gyr old (Figure 4 ). The lack of YSOs in clusters older than 0.4 Gyr implies that the age difference between multiple-generations of stars in these clusters is less than 0.4 Gyr. This is consistent with the maximum age differences of multiple stellar populations derived for clusters in the LMC (Milone et al. 2009). While $\lesssim 0.4$ Gyr age spread is commonly found among intermediateage clusters (Mackey et al. 2008; Goudfrooij et al. 2015; Milone et al. 2009), some clusters with similar age to those in our study are consistent with a single stellar population (Milone et al. 2013; D'Antona et al. 2015) or with relatively small age spread (i.e., 0.08 and 0.15 Gyr; Correnti et al. 2015; Milone et al. 2015). Given the accuracy of age estimation for SCs, these are still consistent with the present results that the age difference can be $\sim 0.1$ Gyr for some clusters. Since accretion of interstellar gas onto clusters does not depend on the ages of clusters, the result strongly suggests that the required fresh gas supply for the second-generation star formation most likely originates from gas ejected by stars inside the clusters. All massive stars (more massive than 8 
$\mathrm{M}_{\odot}$ ) would have already exploded as supernovae in the SCs with ages greater than 0.1 Gyr. The gas ejected from supernovae is highly unlikely to still remain within the clusters. Some intermediate-mass stars in the clusters are currently dying and ejecting gas through their stellar winds. If the ejected gas can be trapped by the gravitational potential of the cluster, star formation from that gas maybe possible.

The current star formation rate of the LMC estimated from 299 YSO candidates is $0.06 \mathrm{M}_{\odot} \mathrm{yr}^{-1}$ (Whitney et al. 2008). This rate is a lower limit because of possibly missing YSO candidates in the observation. If the star formation rate is scaled to 15 YSOs found in the clusters of our study, it corresponds to $0.003 \mathrm{M}_{\odot} \mathrm{yr}^{-1}$. This implies that the total mass of stars formed in SCs over the last 0.1-1 Gyr can be as large as $2.7 \times 10^{6} \mathrm{M}_{\odot}$. This mass is quite significant as compared to the possible total mass of $9.9 \times 10^{6} \mathrm{M}_{\odot}$ for $0.1-1 \mathrm{Gyr}$ old SCs with an assumed ( $V$-band) mass-to-light ratio of 2.8 in the LMC. The large fraction of later generations of stars formed in clusters is comparable to the observed fractions of second-generation stars inferred from the colour-magnitude diagrams for some LMC clusters (Milone et al. 2015). Therefore, the second generation of stars in these clusters could have been formed in the centre regions of their original clusters.

Given that dusty YSOs evolve into optically visible pre-main sequence stars (PMS; De Marchi et al. 2013), the present detection of YSOs implies that there could be PMS stars in the older clusters. Pre-main sequence stars have been discovered for several SCs in the LMC and Small Magellanic Cloud (SMC) using deep HST observations (De Marchi et al. 2013; Gouliermis et al. 2012), though the clusters are younger than those investigated in this paper. These PMS stars are very faint $(V>22 \mathrm{mag})$ and can not be investigated in existing G10 catalogue derived from groundbased observations. If PMS stars were discovered in the old clusters with YSOs, then such findings would present an additional irrefutable evidence for the presence of multiplegeneration of stars in clusters. Since one YSO is detected per SC, the star formation rate is $2.0 \times 10^{-4} \mathrm{M}_{\odot} \mathrm{yr}^{-1}$ in each cluster. This low star formation rate implies that the number of PMS stars in each cluster is much lower than those detected in young clusters with initial star bursts. Nevertheless, it is worthwhile for future observational studies to investigate the numbers and masses of PMS stars in older clusters. Such observations would better constrain the ongoing star formation rates in older clusters.

Our finding also suggests that the gas supply for secondgeneration star formation cannot originate from young massive stars but must be from old AGB stars. The presence of low-luminosity clusters $(V \sim 17 \mathrm{mag})$ in our sample which contains YSOs does not provide support for theoretical predictions of a threshold mass of globular clusters for secondgeneration star formation (D'Ercole et al. 2008; Bekki 2011). It is unclear how low-mass clusters can retain ejecta from AGB stars for further star formation. However, if low-mass clusters interact with the cold gas from molecular clouds, accretion can occur (Pflamm-Altenburg \& Kroupa 2009). Such cold gas accretion might help low-mass SCs to retain some fraction of AGB ejecta, though this process needs to be investigated.

\section{ACKNOWLEDGEMENTS}

This publication makes use of NASA/IPAC archival data and data products from the Parkes telescope and the Australia Telescope Compact Array. The Australia Telescope Compact Array/Parkes radio telescope is part of the Australia Telescope National Facility, which is funded by the Commonwealth of Australia for operation as a National Facility managed by CSIRO. We thank the referee for his/her constructive comments.

\section{REFERENCES}

Bastian N., de Mink S. E., 2009, MNRAS, 398, L11

Bastian N., Cabrera-Ziri I., Davies B., Larsen S. S., 2013, MNRAS, 436, 2852

Bekki K., 2011, MNRAS, 412, 2241

Bekki K., Mackey A. D., 2009, MNRAS, 394, 124

Bica E., Bonatto C., Dutra C. M., Santos J. F. C., 2008, MNRAS, 389,678

Cabrera-Ziri I., et al., 2016, MNRAS, 459, 4218

Correnti M., Goudfrooij P., Puzia T. H., de Mink S. E., 2015, MNRAS, 450, 3054

D'Antona F., Di Criscienzo M., Decressin T., Milone A. P., Vesperini E., Ventura P., 2015, MNRAS, 453, 2637

D'Ercole A., Vesperini E., D'Antona F., McMillan S. L. W., Recchi S., 2008, MNRAS, 391, 825

De Marchi G., Beccari G., Panagia N., 2013, ApJ, 775, 68

Dieball A., Müller H., Grebel E. K., 2002, A\&A, 391, 547

Dunham M. M., et al., 2015, ApJS, 220, 11

Glatt K., Grebel E. K., Koch A., 2010, A\&A, 517, A50

Goudfrooij P., Puzia T. H., Kozhurina-Platais V., Chandar R., 2009, AJ, 137, 4988

Goudfrooij P., et al., 2014, ApJ, 797, 35

Goudfrooij P., Girardi L., Rosenfield P., Bressan A., Marigo P., Correnti M., Puzia T. H., 2015, MNRAS, 450, 1693

Gouliermis D. A., Schmeja S., Dolphin A. E., Gennaro M., Tognelli E., Prada Moroni P. G., 2012, ApJ, 748, 64

Gruendl R. A., Chu Y.-H., 2009, ApJS, 184, 172

Kim S., Dopita M. A., Staveley-Smith L., Bessell M. S., 1999, AJ, 118, 2797

Kim S., Staveley-Smith L., Dopita M. A., Sault R. J., Freeman K. C., Lee Y., Chu Y.-H., 2003, ApJS, 148, 473

Li C., de Grijs R., Deng L., 2014, Nature, 516, 367

Li C., de Grijs R., Deng L., Geller A. M., Xin Y., Hu Y., FaucherGiguère C.-A., 2016, Nature, 529, 502

Mackey A. D., Broby Nielsen P., 2007, MNRAS, 379, 151

Mackey A. D., Broby Nielsen P., Ferguson A. M. N., Richardson J. C., 2008, ApJ, 681, L17

Meixner M., et al., 2006, AJ, 132, 2268

Milone A. P., Bedin L. R., Piotto G., Anderson J., 2009, A\&A, 497, 755

Milone A. P., Bedin L. R., Cassisi S., Piotto G., Anderson J., Pietrinferni A., Buonanno R., 2013, A\&A, 555, A143

Milone A. P., et al., 2015, MNRAS, 450, 3750

Milone A. P., et al., 2016, MNRAS,

Pflamm-Altenburg J., Kroupa P., 2009, MNRAS, 397, 488

Piatti A. E., et al., 2014, A\&A, 570, A74

Piotto G., et al., 2007, ApJ, 661, L53

Piotto G., et al., 2015, AJ, 149, 91

Seale J. P., et al., 2014, AJ, 148, 124

Sewiło M., et al., 2010, A\&A, 518, L73

Whitney B. A., et al., 2008, AJ, 136, 18

Yamaguchi R., Mizuno N., Onishi T., Mizuno A., Fukui Y., 2001, PASJ, 53, 959

Yang W., Bi S., Meng X., Liu Z., 2013, ApJ, 776, 112 


\section{L6 B.-Q. For and K. Bekki}

Zaritsky D., Harris J., Thompson I. B., Grebel E. K., Massey P., 2002, AJ, 123, 855

Zaritsky D., Harris J., Thompson I. B., Grebel E. K., 2004, AJ, 128,1606

van den Bergh S., 2000, The Galaxies of the Local Group. Cambridge

This paper has been typeset from a $\mathrm{T}_{\mathrm{E} X} / \mathrm{LAT}_{\mathrm{EX}}$ file prepared by the author. 